Begegnungen mit Bach 


\title{
Begegnungen mit Bach
}

\author{
Eine Anthologie zugunsten \\ der Berliner Bach-Autographe
}

Herausgegeben von Friedemann Kluge

Bärenreiter

Metzler 
Die Deutsche Bibliothek - CIP-Einheitsaufnahme

Ein Titeldatensatz für diese Publikation ist

bei Der Deutschen Bibliothek erhältlich.

Besuchen Sie uns im Internet:

www.baerenreiter.com

www.metzlerverlag.de

(C) 2002 Springer-Verlag GmbH Deutschland

Ursprünglich erschienen bei Bärenreiter-Verlag Karl Vötterle GmbH \& Co. KG, Kassel Gemeinschaftsausgabe der Verlage Bärenreiter, Kassel,

und J. B. Metsler, Stuttgart und Weimar 2002

ISBN 978-3-476-01910-3 (Metzler)

ISBN 978-3-476-01910-3 ISBN 978-3-476-02867-9 (eBook)

DOI $10.1007 / 978-3-476-02867-9$ 


\section{Inhalt}

Geleitwort 1

Vorwort 3

Dorothee Sölle

Weihnachtsoratorium $\quad 7$

Helmut Schmidt

Musik "zur Recreation des Gemüths" 8

Nikolaus Harnoncourt

Zur ersten Aufführung der Matthäuspassion

durch den Concentus Musicus

Christoph Wolff

Noten, Orgeln und Motetten

Martin Petzoldt

Mein erstes Bach-Erlebnis - auch ein Beitrag zur Aufführungspraxis

Ludger Udolph

"Aber Menschenopfer unerhört«.

Gedanken zum Opfermythos in Bachs Matthäuspassion

Manfred Stolpe

Ein Bach in dürrer Zeit

Christian Geissler (k)

Liebster Gott, wann werd ich sterben

Dieter Zimmer

Der Buscher, Bella-bella-Marie und B-A-C-H

Regine Hildebrandt $(\dagger)$

Vielfalt der Begegnungen:

Von Anna Magdalena bis zu den verschwiegenen Töchtern

Hartmut von Hentig

"Ich bin's, ich sollte büßen" 
Sabine Peters

Ein bewohnbarer Ort?

Fritz Göttler

Bach im Kino

Hans-Klaus Jungheinrich

Bach-Initiation im Tretrad. Die erste Matthäuspassion -

Leiden eines Achtjährigen

Friedemann Kluge

Katzenmusik

Barbara Schlick

Und sang mir die Kehle aus dem Leib

Martin Geck

Sinfonia f-Moll

Astrid Horst

Bach-Kantaten contra Voodoo Chile

Giselher Klebe

Speicheltropfen und Knackgeräusche

Rolf Riehm

Blutkörperchen

Georg Christoph Biller

Heimweh

Ruth Zechlin

Mitten ins Herz

Hartmut Stauder

Dreimal Bach: Tacet - Fortissimo - Echo

Lothar de Maizière

Zeichen der Hoffnung

Krzysztof Meyer

Phänomenale Schönheit

Ludwig Güttler

Sextsprung auf Jehova 
Herbert Hildebrandt

Spannende Entdeckungen

Peter Giersdorf

Kraftspendender Wohllaut

Dietrich Erdmann

Bach, Lebensbegleiter

Heinrich Magirius

Goldrausch der Forsythiabüsche

Ulla Groenewold

Tobe, Welt, und springe!

Peter Maser

Mein erster Bach oder: Weshalb ich niemals Abitur gemacht habe

Margrit Schridde

Ein langer $W e g$

Thomas Gabriel

Multidimensional

Magdalene Hundertmark

Tyrannei und Kirchenbank

Susanne Höbel

Im Konzertsaal

Hans-Joachim Hespos

Musik, die mich weiterträgt

Klaus Th. Götzelt

Bach oder nicht Bach, das ist hier die Frage

Heinz Knobloch

Das dritte Brandenburgische Konzert

Gerald Woehl

Wie im Traum

Maria Kraa

Nie wahrgenommene Klänge

Rainer Lechtenbrink

Ein maßstabsetzendes Hörerlebnis 
Reinhard Grotz

Psalmenpumpe und Himmelskönig

Gottfried Wagner

Warum Bach einer meiner Lieblingskomponisten ist 142

Theo Adam

Mein erster Bach

Siegfried Matthus

Die Tiefe der Musik

Über die Autorinnen und Autoren 


\section{Geleitwort}

B erlin ist keine "genuine" Bach-Stadt, wenn man ausschließlich B Johann Sebastian Bach meint. Das Leben des großen Komponisten weist kaum Bezüge zu diesem Ort auf. Daß Berlin trotzdem zu den bedeutendsten "Bach-Städten " Deutschlands zählt, liegt in der weltweit berühmten Bach-Sammlung der Staatsbibliothek zu Berlin/PreuBischer Kulturbesitz begründet. Sie vereinigt mehr als achtzig Prozent des erhaltenen kompositorischen Nachlasses von Bach: Werkautographe und originale Stimmensätze besonders aus seiner Leipziger Zeit als Thomas-Kantor. Rund dreihundert Einheiten zählend, gehört sie nun dem Weltkulturerbe an. Es befinden sich darunter die großen Werke und Werksammlungen wie die Johannes- und Matthäuspassion, das Weihnachtsoratorium, die h-Moll-Messe, die Brandenburgischen Konzerte, die Kunst der Fuge und der Erste Teil des Wohltemperierten Klaviers, außerdem die meisten autographen Partituren des Kantatenwerks. Flankiert wird diese Sammlung von Werkautographen seiner Söhne und von weiteren Vertretern der Bach-Familie.

Daß sich dieser einzigartige Bestand gerade in Berlin herausbildete, hängt mit den glücklichen Umständen im Vorfeld des Aufbaus einer eigenen Musikabteilung in der ehemals Königlichen Bibliothek zu Berlin zusammen. Mit dem Wandel des allgemeinen Musikgeschmacks im 18. Jahrhundert erlahmte beinahe überall und fast schlagartig das Interesse an Bach und gefährdete die Tradition einer kontinuierlichen Aufführungspraxis. In Berlin war das allerdings anders. Dafür sorgten Carl Philipp Emanuel Bach als Cembalist am Hofe Friedrichs des Großen und sein Bruder Wilhelm Friedemann. Auch wirkten einige Schüler des Vaters in herausragenden Positionen des Berliner Musiklebens. Und die BachVerehrung der Prinzessin Amalia hielt allem Einfluß des zeitgenössischen Musikgeschmacks stand. So besaß die Schwester Friedrichs des Großen unter anderem das Autograph der Brandenburgischen Konzerte.

Die erste bedeutende Bach-Sammlung in Berlin baute die Singakademie zu Berlin auf, besonders ab 1800 unter der Leitung von Carl 
Friedrich Zelter. Ihm gelangen teilweise spektakuläre Erwerbungen, die sich vor allem auf die Nachlässe Carl Philipp Emanuel und Wilhelm Friedemann Bachs stützten. Die Sammeltätigkeit der Institution gipfelte in der denkwürdigen Wiederaufführung der Matthäuspassion von Johann Sebastian Bach durch die Singakademie unter der Leitung von Felix Mendelssohn Bartholdy im Jahre 1829. Diese Aufführung war es, die das Werk Bachs wieder vollständig im musikalischen Bewußtsein der Zeit verankerte. Sie war ein wesentlicher, wenn nicht sogar der Ausgangspunkt für die moderne Entfaltung der Werke des Komponisten.

So bedeutend die zentrale Vereinigung der Quellen in Berlin gerade auch für die historische Quellenbearbeitung ist, so gewaltig ist die konservatorische Aufgabe, vor die sich die Staatsbibliothek gestellt sieht. Da der Komponist meist die "sauren", sehr aggressiven Tinten verwendete, bedroht heute der sogenannte Tintenfraß in teils dramatischer Form die Dokumente, auch in ihrer inhaltlichen Substanz. Die Staatsbibliothek hat sich deshalb zu einer "Generalüberholung " der Bach-Autographe entschlossen und arbeitet dabei mit dem Zentrum für Bucherhaltung in Leipzig zusammen. Alle verfügbaren konservatorischen und restauratorischen Methoden werden angewandt. Verfahren wie die sogenannte Papierspaltung werden inzwischen so gut beherrscht, daß der Zerstörungsprozeß, dem die Quellen ausgesetzt sind, gestoppt werden kann. Darüber hinaus sichert die Farb-Neuverfilmung des gesamten Bestands den Forschern weltweit den Zugriff und die Auseinandersetzung mit dem Material.

Die hochspezialisierte, wissenschaftsgebundene und handwerkliche Arbeit hat, zusammen mit dem notwendigen Transport und der Versicherung, einen hohen Preis. Er bewegt sich im hohen siebenstelligen Bereich und ist kurzfristig und aus eigener Kraft von der Staatsbibliothek nicht aufzubringen. Die dramatische Situation des Bach-Bestands duldet aber keinen Aufschub.

Deshalb hat der Förderverein Freunde der Staatsbibliothek zu Berlin e.V. das Projekt Bach Patronat ins Leben gerufen und bittet derzeit mit verschiedenen Aktionen - unter anderem mit diesem Buch - alle, denen das Werk Bachs etwas bedeutet, um Unterstützung für die große Aufgabe. Das Projekt ist bislang sehr erfolgreich, es fehlen aber immer noch erhebliche Mittel, um den Wettlauf mit der Zeit zu gewinnen.

Prof. Dr. h. c. Klaus-Dieter Lehmann Präsident der Stiftung Preußischer Kulturbesitz 


\section{Vorwort}

In der kulturellen Gemengelage Mitteleuropas ist es eher unwahr1 scheinlich, daß ein erwachsener Mensch noch nie einen Ton von Mozart, Beethoven oder eben: Bach gehört hätte - und sei es zufällig, als Hintergrundgeräusch oder aus einem benachbarten, nicht im Eigenbesitz befindlichen Tonträger. Eine solche Begegnung verlangt auch stets eine Stellungnahme: Wer ausschließlich auf alpenländische Volksklänge abonniert oder Rezipient hammerschwerer Techno-Geräusche ist, wird - etwa durch Schließen des Fensters - die Quelle solcher Musik augenblicklich versiegen lassen. Nichtsdestoweniger aber hat eine Begegnung stattgefunden: Man hat ein paar Takte lang Mozart, Beethoven oder Bach gehört (in der Regel natürlich, ohne sie den Genannten zuordnen zu können oder dieses auch nur zu wollen) - und eine Entscheidung getroffen.

Oder aber man läßt sich auf diese Musik ein, um erst nach dem Hören ein Urteil zu fällen, das auch dann immer noch ein ablehnendes sein kann, weil die eigenen musikalischen Präferenzen anderswo beheimatet sind oder weil die musikalische Vorbildung nicht ausreicht. Jeder nicht chronisch hörgeschädigte Mitteleuropäer wird also irgendwann einmal eine Begegnung mit Mozart oder Beethoven gehabt haben. Oder mit $\mathrm{Bach} . \mathrm{Ob}$ er selbst sich an dieses Erlebnis erinnert, ist eine andere Frage. $\mathrm{Ob}$ es überhaupt ein erinnernswertes Erlebnis war, eine weitere.

Eine andere Art von "Erinnerung« begegnet uns in den Gegenständen der Material- oder Sachkultur, wie sie uns z.B. in den erhaltenen Handschriften Johann Sebastian Bachs als Erinnerungsstücke überliefert sind. Diese der Nachwelt zu erhalten und als "Kulturerbe« zu einem Bestandteil ihrer Erinnerung werden zu lassen, ist die Aufgabe derer, in deren Obhut sich diese Kostbarkeiten befinden.

Mit rund 300 Autographen ist der Bestand der auf uns überkommenen Original-Handschriften Johann Sebastian Bachs erstaunlich umfangreich. Ihr größter Teil ging zunächst in den Besitz seines Sohnes Carl Philipp Emanuel (1714-1788) über, dessen Nachlaß wiederum 
der bedeutende Musikaliensammler Georg Johann Daniel Poelchau (1773-1836) erwarb. Auf dem Umweg über die damalige Königliche Bibliothek zu Berlin bzw. über das Archiv der Berliner Singakademie des Goethe-Freundes Carl Friedrich Zelter (1758-1832) gelangten bis heute rund $80 \%$ der in öffentlichem Besitz befindlichen Bach-Handschriften in den Bestand der Berliner Staatsbibliothek. Unter ihnen befinden sich beispielsweise der erste Teil des Wohltemperierten Klaviers, Die Kunst der Fuge, die Brandenburgischen Konzerte, die Orchesterouvertüren Nr. 2 und 3, die h-Moll-Messe, das Weihnachtsoratorium, die erhalten gebliebenen Passionen, zahlreiche Kantaten, Orgelwerke und manch andere unersetzliche Bachsche Preziose. Die rund 135.000 Seiten umfassende Bach-Sammlung der Staatsbibliothek enthält überdies zahlreiche Autographen und Abschriften von den Kompositionen der Söhne und anderer Familienmitglieder, aber auch Werke von Bach-Schülern und Zeitgenossen.

Die meisten dieser bis zu 300 Jahre alten Handschriften befinden sich jedoch in großer Gefahr: Die von Bach vorwiegend verwendete säurehaltige Tinte erweist sich heute als eine Zeitbombe; insbesondere seine Autographen sind durch den sogenannten Tintenfraß gefährdet. Mittlerweile haben ebenso erfolgreiche wie - leider - sehr kostspielige Restaurierungsmaßnahmen begonnen, um den Handschriftenbestand der Nachwelt zu erhalten. Insgesamt werden dafür mindestens zwei Millionen Mark benötigt. Zur Finanzierung dieses Vorhabens wurde das Bach Patronat der Freunde der Staatsbibliothek gegründet, das sich über jede, auch die kleinste Spende freut.

Als eine indirekte Spende versteht sich vorliegendes Buch. Die daran beteiligten Autorinnen und Autoren verzichten auf ihre Honorare, die der Restaurierung und Pflege des Bach-Handschriftenbestandes der Berliner Staatsbibliothek zugute kommen.

Insgesamt fanden sich 46 Autorinnen und Autoren aus den unterschiedlichsten gesellschaftlichen Schichten dazu bereit, über ihre - vielfach ersten - Begegnungen mit der Musik Johann Sebastian Bachs zu reflektieren. Daß der Schwerpunkt hier bei solchen Persönlichkeiten liegt, die auch beruflich mit Musik zu tun haben, kann nicht verwundern: Prominente Sängerinnen und Sänger, Dirigenten, Komponisten, Instrumentalsolisten, Musikwissenschaftler, Jazz-Musiker und sogar ein Orgelbauer konnten zur Mitwirkung an dieser Anthologie gewonnen werden. 
Die sich aus der soziologischen "Streubreite« der Verfasser erklärende Heterogenität der Beiträge ist nicht Mangel, sondern Reichtum, zeigt sie doch, in welch unterschiedlicher Weise man sich dem Thema Bach nähern kann: Musikologische oder sogar textologische Ansätze sind ebenso vertreten wie das von den meisten Autoren bevorzugte chronikalische Berichten, der humoristische Rückblick auf ein BachErlebnis hat ebenso seinen Platz gefunden wie die literarische Stilisierung, die lyrische Annäherung ist ebenso vorhanden wie die nüchternprosaische.

"Bachs Kompositionen sind im schönsten und reinsten Sinne deutsche Musik." Dieses in einem der Beiträge gezogene Fazit hält sich fern jedweder dumpfen Deutschtümelei. Aber es ist richtig: Eine deutsche Musik ist es, weil sie vor allem in deutschen Elternhäusern buchstäblich von der Wiege bis zur Bahre gepflegt wurde und bisweilen auch noch wird. Insbesondere den kirchenverbundenen Menschen (im übrigen: beider großer Konfessionen) begleitet Bach mit seinen Chorälen, Kantaten, Passionen, dem Weihnachtsoratorium, den Orgelwerken, dem Magnificat oder der Messe in h-Moll einerseits durch das komplette Kirchenjahr, andererseits durch das Leben und sogar im Sterben, oder, wie es in einem anderen Beitrag heißt: "Bach stand [...] am Anfang, und er wird auch in irgendeiner Form am Ende stehen."

"Deutsche Musik« konnotiert aber auch noch etwas anderes: Gerade und besonders im vergangenen, kriegs- und krisengeschüttelten Jahrhundert war Bach ein (gewiß höchst unfreiwilliger) Begleiter insbesondere der deutschen Historie und ihrer Auswirkungen auf den Einzelnen. In den hier vorliegenden Darstellungen steht Bach nicht selten in einer assoziativen Verbindung mit Nazi-Deutschland und dem Zweiten Weltkrieg oder mit der über vier Jahrzehnte bestehenden deutschen Teilung. Es sind dies Geschichten, wie sie sich eben nur in Deutschland zutragen konnten - oder in jenen Ländern, die das Unglück hatten, im Verlauf zweier Weltkriege in den verheerenden Sog deutscher Großmannssucht zu geraten. Davon weiß der polnische Komponist Krzysztof Meyer - nicht nur seinem Namen nach ein "Grenzgänger« - zu berichten, wenn er sich daran erinnert, wie schwierig es im Nachkriegspolen war, sich mit Bach-Noten zu versorgen.

Eine Auseinandersetzung mit der Bachschen Musik als solcher, also ohne historische Assoziationen, findet in anderen Beiträgen statt, wieder andere rücken pädagogische Aspekte in den Vordergrund oder thematisieren die Verwendung Bachscher Kompositionen im Film. 
Allen, die an dem Buch mitgewirkt haben, sage ich meinen herzlichen Dank, insbesondere natürlich den Autorinnen und Autoren, die sich (oftmals unter erheblichem Zeitdruck) der Mühe unterzogen haben, einen eigenen Beitrag zu verfassen. Mein Dank gilt aber auch den vielen Persönlichkeiten, die, ohne selbst über eine Erinnerung an ihr erstes Bach-Erlebnis zu verfügen oder eine solche plastisch werden lassen zu können, das Werden des Buches mit ihren guten Wünschen begleitet haben. Ein weiterer Dank geht nach Lienen-Kattenvenne bei Osnabrück, wo mir mein Freund, der Buchhändler Helmut Ruck, ein weiteres Mal mit hilfreichem Rat und wertvoller Recherche weitergeholfen hat. In ganz besonderer Weise möchte ich endlich der Lektorin des Bärenreiter-Verlages, Frau Dr. Jutta Schmoll-Barthel, und dem Vorsitzenden der Neuen Bachgesellschaft, Herrn Prof. Dr. Martin Petzoldt, danken. Ihrem Zuspruch, Engagement und Verhandlungsgeschick ist es zu danken, daß dieses Buch erscheinen kann. Ein reiches Leserinteresse sei der Mühe Lohn!

Berlin, im Dezember 2001

Friedemann Kluge 